\title{
Distal Tactile Feedback for Text Entry on Tabletop Computers
}

\author{
Christopher McAdam and Stephen Brewster \\ Glasgow Interactive Systems Group, Department of Computing Science \\ University of Glasgow, Glasgow G12 8QQ, UK \\ +441413304966 \\ \{chrism, stephen\}@dcs.gla.ac.uk www.dcs.gla.ac.uk/ stephen
}

\begin{abstract}
In this paper we present an initial study into the feasibility of using a mobile phone as a personal tactile display when interacting with a tabletop computer. There has been an increase in recent years in large touchscreen computers that use soft keyboards for text input. Text entry performance on such keyboards can be poor due to the lack of tactile feedback from the keys. Our approach is to use the vibration motor in a user's mobile phone to provide personal haptic feedback for interactions with the touchscreen computer. We ran an experiment to compare text entry on a touchscreen device with the tactile feedback being presented at different distal locations on the body (locations at which a user might keep a mobile device. The conditions were: no tactile feedback, feedback directly on the device, feedback at the wrist, upper arm, chest, belt and trouser pocket). The results showed that distal tactile feedback significantly increased text entry rates when presented to the wrist and upper arm. This was not at the expense of a reduction in text entry accuracy. This shows that the concept of presenting tactile feedback on a user's phone is an effective one and can improve interaction and text entry on tabletop computers.
\end{abstract}

\section{Categories and Subject Descriptors}

H.5.2 [User Interfaces]: Haptic I/O

\section{General Terms}

Human Factors.

\section{Keywords}

Tactile feedback, tabletop interaction, text entry, mobile phone

\section{INTRODUCTION}

Tabletop computing is an area which has seen an increase both in research and in public awareness in recent years, due in part to Han's work with FTIR [8] and many companies now make units available to the public $[1,2,3,14]$ (see Figure 1). Tabletops also allow for novel forms of interaction, such as gesturing and the use of tangible controllers [15] placed on the surface of the table. These tabletops are typified by their inclusion of di-

(c) The Author 2009.

Published by the British Computer Society rect finger-based multitouch input and a 360 degree user experience. This configuration means that users should be able to use the table without restriction no matter where they are positioned around it. As such, it makes little sense for such devices to have physical input devices like mice or keyboards attached to them. A soft keyboard, which can be displayed on screen when required and oriented to suit the user, is the most common replacement for physical keyboards in tabletops. Text entry for tabletop computers is important: users need to be able to type messages, URLs, label photos, etc., so we need to ensure that text entry performance is good otherwise the whole user experience will be frustrating.

While the soft keyboards used on these devices are modelled on their physical counterparts they are not a direct match. For example they can be resized to suit the user or application. However, these keyboards do not provide the same tactile feedback as their physical counterparts. The surface of the tabletop is smooth so users cannot actually feel the keys (or buttons or other widgets). Without this feedback, the user relies on visual cues while typing to ensure accuracy.

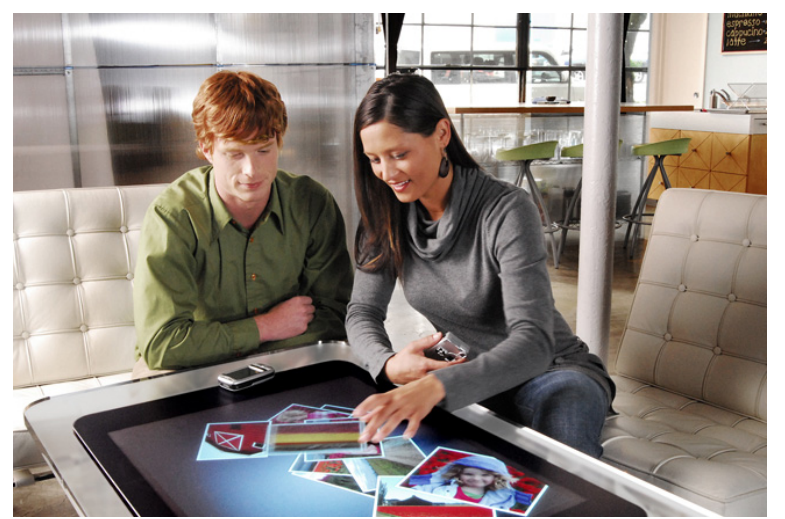

Figure 1: Example of Tabletop Computer usage [taken from www.microsoft.com/presspass/presskits/surfacecomputing/ gallery.mspx].

Our previous work [13] has shown that typing performance can be reduced on touchscreens where there is no tactile feedback, in their case on touchscreen mobile phones. Our solution used tactile feedback from the vibration motor in the phone to replace the missing feedback from a physical keyboard. The aim of the research here was to see if the same issues occurred with tabletop soft keyboards and if additional tactile feedback could help overcome them.

The addition of tactile actuators to a tabletop computer is difficult as the size of the screen would require a powerful vibration motor. There would also be issues of interference as other users on the table would also feel the feedback on typing, potentially causing annoyance or confusion. Our solution is to use each 
user's mobile phone as a private tactile display. By this we mean the phone will be used to deliver information to the user in a non-visual way utilising the phone's vibration motor.

Mobile phones are now extremely common, with $60 \%$ of the world's population owning one [30]. This means that many tabletop users will have a phone with them when they come to use the tabletop computer. We can take advantage of this for interaction. Mobile phones are versatile devices, and are capable of generating visual, tactile and audio feedback [10], providing Internet access, Bluetooth and IR connectivity, and increasingly models are featuring sensors such as accelerometers and magnetometers. While some of these features are currently available only on higher-end models, the ability to produce tactile feedback is standard on the vast majority of devices (e.g. the Nokia 1200, the most basic phone on the Nokia UK website, features vibration alerts). By pairing the device with the table, the phone will be able to act as a user's personal visual, audio and haptic display and input device. We propose to utilize the tactile actuator in the mobile phone to provide feedback for text entry on the tabletop.

This paper presents an initial study into the feasibility of using tactile feedback in this way. We describe an experiment into the effectiveness of tactile feedback for typing on the tabletop to see if we can gain the same performance advantages shown by Hoggan in our previous work [13]. An important issue is that the feedback will not be on the user's hands when he/she is typing (as it was in Hoggan et al.'s study). The phone is more likely to be in a pocket as the hands need to be free, so the feedback will be distal to the interaction on the tabletop and therefore may not be perceptually integrated with the typing actions. Our aim is to determine if such feedback is beneficial to the user, and to see how its effects are altered depending on the location on the body to which the feedback is delivered.

\section{BACKGROUND}

Current active areas of research in tabletop computing are focused heavily on the issues of territory, interaction and orientation $[19,25,31]$. However, there are as yet few studies into the general usage of tabletop computers, partly due to the relatively recent development of readily available units. Ryall et al. [24] observed that when first presented with a direct-touch tabletop users tend towards single finger interactions, but once informed are happy to experiment with multitouch interactions. Ryall also notes that the finger can occlude what is being pressed, necessitating the display of a label offset from the point of contact, such as is found in the Apple iPhone.

Wigdor et al. [32] performed a 13 month long study into an individual's use of a direct touch table as his primary computer, and compared that with his usage of a laptop during the same period of time. An analysis of emails typed on both machines found that the length of the messages was significantly longer when created on the tabletop. While the participant stated that "a reasonable person would not use an on-screen keyboard", he did force himself to use it and did enter more text.

Hinrichs [12] notes that little attention has been paid to the development of text entry methods for tabletop displays, but suggests that the unique traits of tabletop displays demand unique text entry methods that differ from the standard physical keyboard. While he describes multiple different text entry options, he does not provide a recommendation on which one would ultimately perform best. We therefore decided to use the standard QWERTY keyboard layout, as it is most commonly used.
Ryall et al.'s [24] observations on text input on tabletop computers lead them to say that "text input is particularly challenging", and that "bare fingers are insufficient for text input". It is mentioned that alternative entry methods, such as Graffiti-style input are not sufficient for large amounts of text, and that the soft keyboard is a "feasible, but tedious solution". The potential use of a physical keyboard is raised in Wigdor et al.'s study, and while it is feasible in the predominantly single user environment present in this study, it is not a solution that will scale as the number of users increases. This previous research suggests that there are significant problems with the standard onscreen keyboards provided for tabletop computers. Either new methods of input need to be found or the keyboards need to be improved to increase text entry performance. Our research is investigating the latter as we believe that keyboards will still be necessary for input in one form or another for a significant time to come.

There have been studies of the design of touchscreen displays, the size of keys [26] and alternative key layouts [18] which provide basic parameters for the design of tabletop keyboards. However, none of these have used tactile feedback which may improve typing performance. There has been research into providing tactile feedback for touchscreen devices, but it has primarily taken place in the context of mobile devices [22] [29] [16]. In our own previous work [13] we performed a study into the effectiveness of adding tactile feedback to mobile touchscreen devices for improving finger-based text entry. We looked at providing specialized tactile feedback to represent the different stages of pressing a physical key - fingertip-over, fingertip-click and fingertip-slip. We compared a mobile phone with a physical keypad, a standard touchscreen phone and the standard touchscreen phone with extra tactile feedback presented through the internal vibration motor. We found the addition of such tactile feedback to the touchscreen significantly improved finger-based text entry, bringing it close to the performance of the real physical phone keypad. In a second experiment, it was found that using higher specification tactile actuators could improve performance even further. Two actuators were placed on the back of a PDA, which allowed for spatial location to be incorporated into the feedback to better give an impression to the user of which button was causing the feedback.

There has been less research performed in the area of multi-user feedback; an important topic because in an initial study of the Ubitable [6] it was found that the auditory feedback used to indicate errors confused the users, as they were unable to tell which user had caused the sound. Ringel Morris et al. [23] found in their work on using individual audio channels for single display groupware that such a system does not impede group communication and may support group interaction in a positive way. In Hancock's [9] work on non-speech auditory feedback, the users were spread around the table and each had a speaker providing their own audio feedback. Again, this is not a feasible solution as the number of users increases, and if the users are grouped around one of the speakers then they will end up sharing the feedback provided.

Brewster [4] has studied the use of audio feedback for touchscreens. Results showed that performance could be improved when audio feedback was given on button down, button up and slip-off. Audio would be a good solution for tabletop computers as the table could easily include a speaker, but the same problems with the Hancock study would apply. An alternative would be to use the mobile phone speaker for a private audio display. If the user wore an earpiece then the others around the 
table would not hear the feedback. This is possible but, depending on the configuration of the earpiece this may impede the communication with others around the table [23]. Tactile feedback is private as only the user holding the phone would feel it and it would not affect communication with other users.

There have been several studies that looked at using mobile phones in conjunction with wall displays, which is of interest as wall displays and tabletops have several characteristics in common. Hardy et al [10] examined using a NFC enabled mobile phone to both send photos to a public display and also to download photos from the display. McCarthy et al. [20] developed a proactive display that changed the content it displayed by detecting nearby people using their Bluetooth phones. Silfverberg at al. [27] performed a study of controlling the cursor on a public display using the joystick on a handheld device. Typically the phone is used as an input to the wall display; as such our work is unusual in that the phone is being used as an output device for the tabletop computer.

\section{EXPERIMENT}

This paper presents an experiment investigating text entry on a touchscreen device with and without tactile feedback. The goal is to explore the effects of delivering the tactile feedback from keyboard events to see if performance can be improved, and to see how the performance is effected by altering the location on the body where that the tactile feedback is presented.

The Samsung Q1 Ultra UMPC was used for the experiment as it has a large touchscreen (7" widescreen, 1024x600 resolution) that can display a soft keyboard of a similar size to that which would be used on a tabletop computer. It is large enough for comfortable one handed finger-based text entry. While this device is smaller than the tabletop systems we are using it to emulate, as we are focused only on the task of text entry, a full sized tabletop display was not required to test our hypotheses. Only a small area of a tabletop display would be required to perform text entry, which we are able to reproduce on the touchscreen of the UMPC. The interactions that the user would use on a tabletop - finger presses - are the same as those on the UMPC. Additionally, while our primary interest is in tabletop displays, the benefits of such distal tactile feedback should apply to all touchscreen devices, not just tabletop computers. This device also allowed us to run exactly the same code that we used in our previous experiment [13], so we could make a direct comparison with our results on mobile touchscreen interactions.

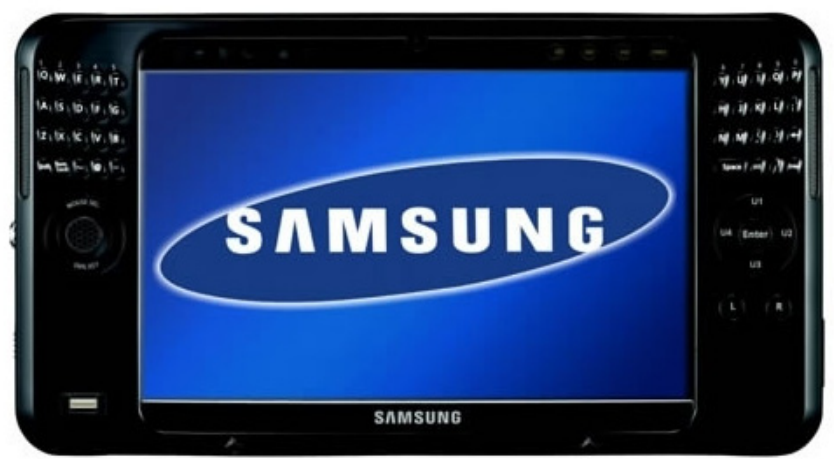

Figure 2: Samsung Q1 Ultra, a touchscreen UMPC (www.samsung.com).

As the Samsung device does not have built-in tactile feedback this was added using a C2 Tactor from Engineering Acoustics Inc (www.eaiinfo.com). The $\mathrm{C} 2$ is a small linear actuator, which is designed specifically to provide a lightweight equivalent to large laboratory-based linear actuators. The contactor in the $\mathrm{C} 2$ is the moving mass itself, which is mounted above the housing and pre-loaded against the skin. This provides localized feedback as only the contact point (the silver dot) vibrates instead of the whole surrounding (see Figure 3). The C2 is resonant at $250 \mathrm{~Hz}$, the frequency of highest sensitivity in the skin [21].

The $\mathrm{C} 2$ is a higher specification tactile actuator than the rotational motors that are typically found in most mobile phones, and by using it we are able to generate higher quality tactile cues. It is also small and light, making it easy to attach to the different locations on the body where we wished to deliver the feedback. This actuator has been used in much previous research and was used by Hoggan in our touchscreen text entry study [13].

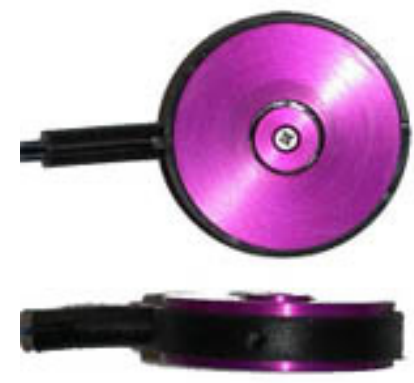

Figure 3: C2 linear actuator from Engineering Acoustics Inc.

\subsection{Feedback Design}

A standard QWERTY touchscreen keyboard was created for use on the Samsung (as can be seen in Figure 4). This keyboard has keys that are $90 \times 80$ pixels in size $\left(1.9 \mathrm{~cm}^{2}\right)$, with a 6 pixel gap between them. Such a keyboard is of a similar size to one that would be used on a tabletop computer.

This experiment re-used the successful tactile feedback from our earlier work [13] in mobile devices. In that study, a set of Tactons [5] was used to represent different keyboard events such as fingertip-over, fingertip-click and fingertip-slip. We replicated these events and Tactons for our experiment to allow our results to be directly comparable to those from our mobile text entry study.

The fingertip-over event allows users to know when they are on a key or moving between keys. When this event is triggered, a 1-beat smooth $300 \mathrm{~ms}$ Tacton is presented. The fingertip-click event signals that a button has been pressed. This is represented by a sharp, 1-beat $30 \mathrm{~ms}$ Tacton. When a finger moves over the edge of any of the buttons on screen, the fingertip-slip event is triggered. The Tacton used here is a 3 -beat rough $500 \mathrm{~ms}$ Tacton is presented.

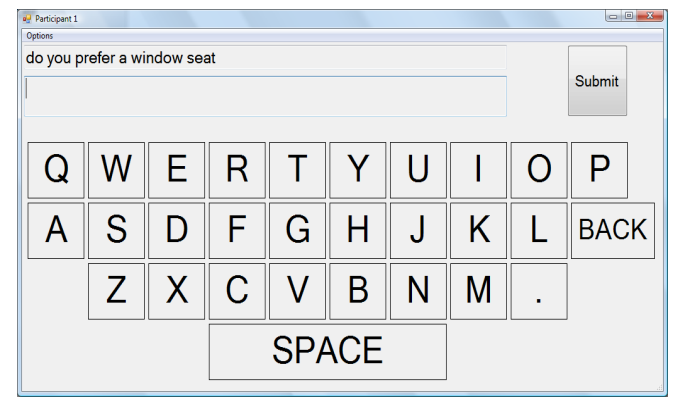

Figure 4: Screenshot of the experimental interface. 
There are additional tactile cues present to help the user navigate the keyboard. In physical keyboards, the home keys of ' $F$ ' and ' $\mathrm{J}$ ' often have raised edges. A Tacton is used here to recreate that when the fingertip-over event is triggered for these keys. The Tacton is a 1 -beat $300 \mathrm{~ms}$ amplitude modulated $250 \mathrm{~Hz}$ sine wave, which feels rough. Home keys are designed to provide tactile feedback to typists so that they do not need to look down at the keyboard, and as such this feedback may not be necessary in this experiment but they are included for both completeness and to ensure as much similarity with Hoggan's experiment as possible.

\subsection{Methodology}

We designed an experiment to investigate the effects of delivering tactile feedback to different body locations while typing on a touchscreen. The experiment compared user performance on a typical touchscreen device with no tactile feedback, to the same device with tactile feedback added, and to the same device but with the tactile feedback delivered to different locations on the body (wrist, upper arm, chest, belt, trouser pocket; shown in Figure 5). As the aim of our work was for the feedback to be delivered from a mobile phone on the user's person, these locations were selected as they provided a mixture of locations in which a phone is likely to be carried (in the trouser pocket, on a belt clip, in a shirt chest pocket), locations that other devices are commonly found (a strap on the upper arm is often used to hold an MP3 player) and locations are of interest for other reasons: the wrist location would mimic a bracelet or watch which included vibration feedback (e.g., www.lmtechnologies.com/ci/index.php? /products/details/38). The 'on device' and no feedback conditions were controls as they replicated those used by Hoggan in our earlier study. Using the same tactile device at each location avoided any differences between different types of actuators from different types of mobile devices.

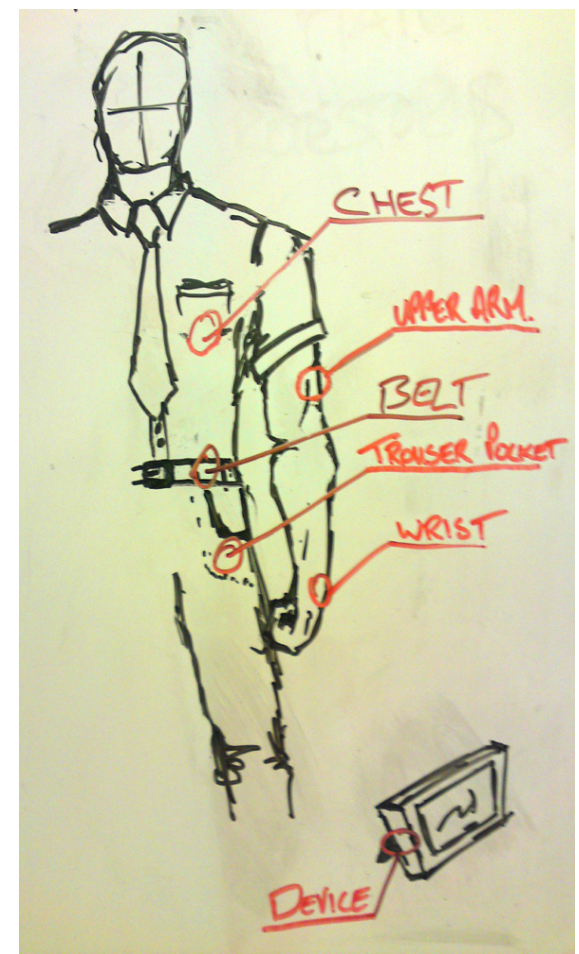

Figure 5: Early design sketch showing tactile feedback locations.
The C2 was attached to the body in different ways depending on the location: a tubigrip support bandage was used for the upper arm and wrist (see Figure 6); the $\mathrm{C} 2$ was attached to the inside of a Polar heart rate monitor strap for the chest condition (as we needed to ensure that we could simulate a shirt chest pocket even if a participant did not have such a pocket in the clothes worn to the experiment); it was attached to the inside of a belt for the waist condition; and for the trouser pocket condition it was placed inside the front right pocket. For the 'on device' condition the $\mathrm{C} 2$ was attached to the back of the device so that the participants would touch the device with their nontyping hands in such a way to ensure that the contact point of the $\mathrm{C} 2$ was against the hand.

We drove the actuator from the Samsung device's headphone socket as shown in Figure 6. We used an audio amplifier to allow the participants to easily change the intensity of vibration to a comfortable level. Users typed using their dominant hand.

The experimental hypotheses were as follows:

1. Tactile feedback will improve speed and accuracy of text entry on touchscreen keyboards;

2. The different body locations used to deliver the tactile feedback will produce differing speed and accuracy.

We used a within-subjects design where the conditions were:

1. Standard touchscreen device with no tactile feedback $(\mathrm{Cn})$;

2. Standard touchscreen device with added tactile feedback on the device (Cd) (as in Hoggan et al. 's study feedback was presented via the $\mathrm{C} 2$ actuator on the back of the device);

3. Standard touchscreen device with tactile feedback added at the wrist $(\mathrm{Cw})$;

4. Standard touchscreen device with tactile feedback added at the upper arm $(\mathrm{Ca})$;

5. Standard touchscreen device with tactile feedback added at the chest $(\mathrm{Cc})$;

6. Standard touchscreen device with tactile feedback added at the waist via a belt $(\mathrm{Cb})$;

7. Standard touchscreen device with tactile feedback added at the front trouser pocket $(\mathrm{Cp})$.

The phrase set used in this experiment was from MacKenzie [17] which has been used successfully in several other studies $[13,18,28]$. It is a 500 -phrase set with no punctuation marks and no uppercase letters. Due to time constraints on our experimental design it was not possible to use the full set of 500 phrases. Instead, a random set of 25 phrases was selected for each condition. All conditions were tested in a static lab environment. The order of conditions was randomized to avoid order effects.

\subsection{Experimental Design}

Sixteen participants took part in this experiment. All participants were students or staff at the University with an age range of 18-33 years. There were six female and ten male participants. One of the participants was left-handed. All participants were seated during the experiment. None had used a tabletop computer before. 
Participants were shown a phrase and asked to memorize it, and then type it in using the keyboard for each condition. They were asked to type as quickly and accurately as possible. There was no noticeable lag between the participant pressing the screen and receiving the tactile feedback. The interface is shown in Figure 4. Each phrase was shown at the top of the screen until the participant began typing, at which point the phrase disappeared. This method sits in between text creation and text copy. Text creation (where users come up with their own phrases) is more realistic but is more difficult to use as errors cannot be easily detected. Text copy is not realistic as it is not a common reason for typing text on a tabletop. The method used in this experiment was not text creation, but the participants were not copying text directly onto the device either, making it a slightly more realistic scenario. This was the approach taken in our earlier study [13] so we copied it to allow us to compare our results to the previous ones. Timing began when the participants hit the first key and stopped when they hit 'Submit'. They moved onto the next task regardless whether the current task was correct or not.

The UMPC used in the study was stood at an angle (as shown in Figure 6). This is different from the horizontal position often found in tabletop computers, where we ultimately wish to apply our work. It has been shown by Forlines et.al [7] that differences in the contact area between the finger and the surface can result in different error rates but this only becomes important when trying to reach distant objects. In our setup the keyboard was always at a fixed, close distance to the participant, so this would not cause any issues and the results will apply equally to horizontal surfaces. Having the table at an angle also allowed for us to include the tactile feedback on the back of the device in one of the conditions.

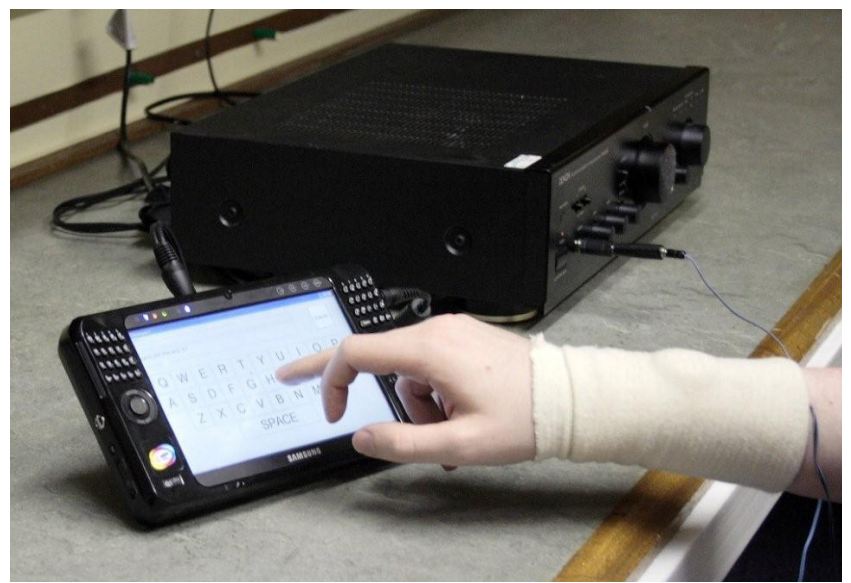

Figure 6: Experimental setup. In this condition $(\mathrm{Cw})$ the $\mathbf{C 2}$ is placed on the wrist using a tubigrip bandage.

A training period was given for each condition to familiarize the user with the interface and the feedback that was being delivered. The training period for each condition consisted of the user entering a maximum of ten test phrases. Participants were able to alter the strength of the tactile feedback to ensure that they could both feel it and that no discomfort was caused. The users altered the strength so that they perceived the intensity as being similar across all body locations. The dependant variables measured in the experiment were speed, accuracy, keystrokes per character (KSPC) and subjective workload (using the NASA TLX workload assessment [11]). In total, participants entered 25 phrases in 7 conditions for a total of 175 phrases entered, plus a minimum 35 phrases from training.

\subsection{Results}

\subsubsection{Accuracy}

Figure 7 shows the accuracy of text input in each of the different conditions. The box plot shows the following: the blue area indicates the $25^{\text {th }}$ percentile up to the median; the red area is from the median up to the $75^{\text {th }}$ percentile; the ends of the lower and upper whiskers show the minimum and maximum values respectively.

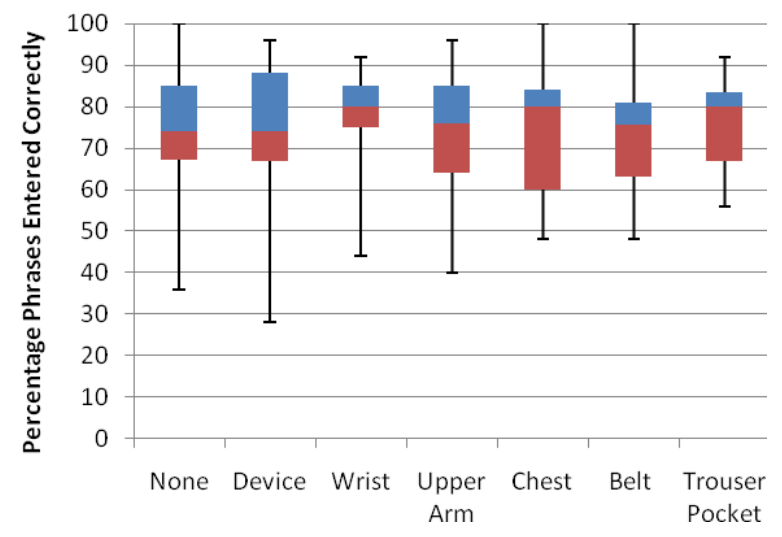

Figure 7: Percentage of phrases entered correctly for each feedback location.

A one-way ANOVA was performed on the mean number of correct phrases entered, comparing the effects of the location where the feedback was delivered. In this case a phrase is considered to be correct if the submitted phrase matched the given phrase at the point when the participant pressed 'Submit', regardless of whether corrections were made or not. There was no main effect for feedback location $(F(6,90)=0.415, \quad p<0.867)$. This suggests that the location of the tactile feedback may not have had an effect on typing performance in this case.

\subsubsection{Keystrokes Per Character (KSPC)}

The number of keystrokes per character is a measure of the number of keystrokes that are required, on average, to produce a character of text for a given text entry technique for a given language, with the ideal being 1 [28]. KSPC was recorded for each condition in the experiment. Given that accuracy scores were based on whether or not the submitted phrase matched the given phrase exactly and did not include corrections as errors, KSPC was recorded in order to examine how many corrections users had to make before submitting a correct phrase. The $\mathrm{KSPC}$ for each location is shown in Figure 8.

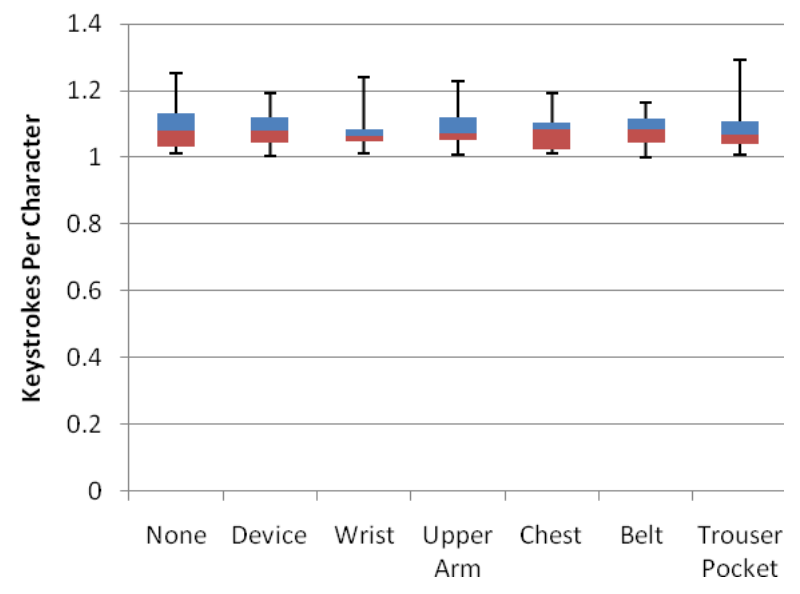

Figure 8: KSPC for each feedback location. 
A one-way ANOVA was performed on the mean number of KSPC, comparing the effects of the location where the feedback was delivered. There was no main effect for feedback location $(F(6,90)=0.697, p<0.652)$. This again suggests that the location of the tactile feedback may not have had an effect on text entry.

\subsubsection{Words per Minute}

Figure 9 shows the words per minute text entry rates for each condition. A one-way ANOVA showed a significant main effect for feedback location $(\mathrm{F}(6,90)=3.38 \mathrm{p}<0.004)$. Post hoc Tukey HSD tests showed the following significant differences (those not presented were not significant).

- $\mathrm{Cw}$ better than $\mathrm{Cn}: \mathrm{p}<0.001$

- Ca better than Cn: $\mathrm{p}<0.005$

- $\mathrm{Cw}$ better than $\mathrm{Cp}: \mathrm{p}<0.01$

- Ca better than Cc: $\mathrm{p}<0.01$

- $\quad$ Ca better than $\mathrm{Cb}: \mathrm{p}<0.02$

These results partially confirm our two hypotheses. We found a significant effect in terms of text entry error rates and also that different body locations performed differently in terms of text entry rate, with upper arm and wrist performing the best of all.

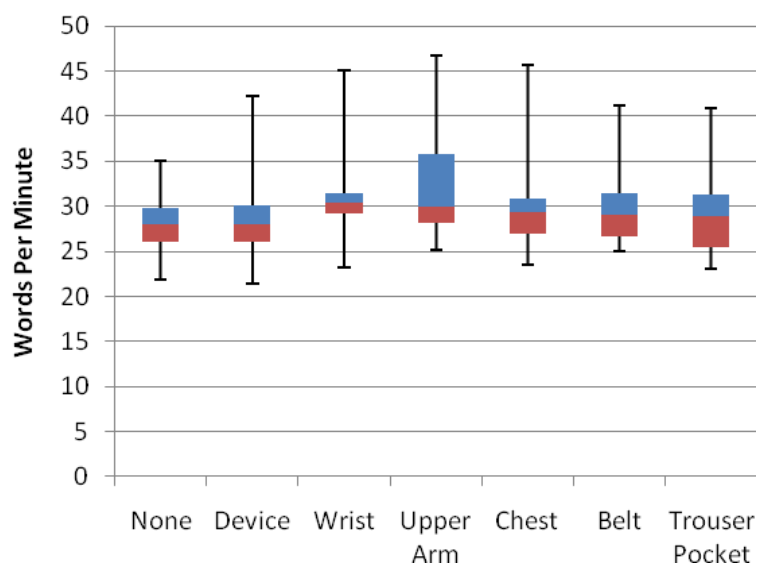

Figure 9: Words per minute for each feedback location.

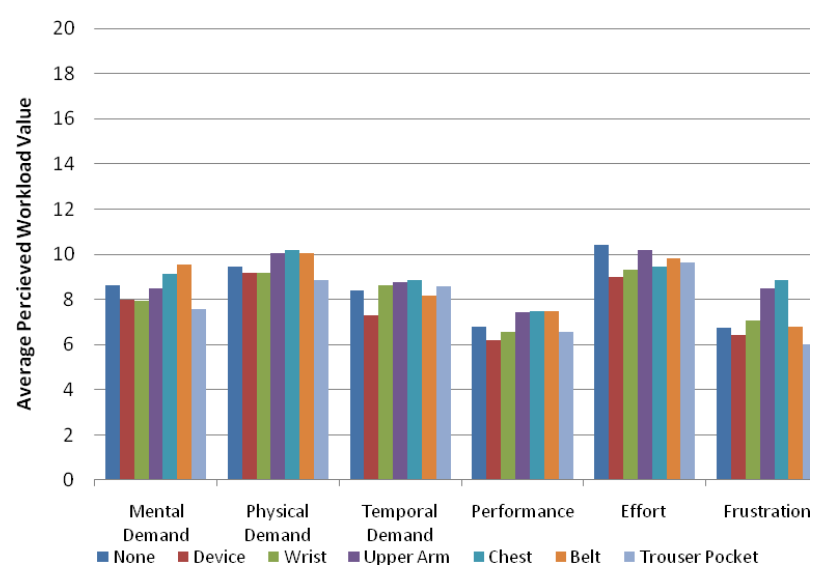

Figure 10: Average NASA TLX scores for each condition.

\subsubsection{Subjective Workload}

The results of the NASA TLX [11] questionnaires are shown in Figure 10. A one way ANOVA on overall workload showed no main effect $(F(6,66)=1.01 \mathrm{p}<0.428)$. This suggests that tactile feedback may not have an effect on the workload of typing on a tabletop computer.

\section{DISCUSSION AND CONCLUSIONS}

Our first hypothesis was that distal tactile feedback would improve the speed and accuracy of text entry, which has been partially supported by the results. We found no significant effects for accuracy or KSPC, suggesting that the tactile feedback does not help in this case. We did find significant effects in terms of words per minute, with users able to type faster (but not at the expense of being more error prone). This is an important finding as text entry rates on soft keyboards are lower than for real keyboards and anything that can be done to improve speed (without compromising accuracy) will be beneficial. It is difficult to add tactile feedback to tabletop computers due to their large and heavy screens. They would need large vibration motors to move them. Using a mobile phone with a vibration motor (or a vibration capable watch/bracelet) connected to the tabletop computer via Bluetooth is a cheap alternative and also avoids the problem of broadcasting the tactile feedback to all users when it is only meant for the person typing (if broadcast to all was needed then a vibrate message could be sent to all of the users' phones via Bluetooth).

It is interesting to consider the reasons why we did not find benefits for accuracy or KSPC. We hypothesise that the benefits of tactile feedback were reduced due to the size of the keyboard. Fitts' Law shows that as the size of targets increases they become easier to hit. Therefore the size of the keys on our keyboard may have made them so easy to hit that the tactile feedback was not needed. In our previous text entry study [13], we found a reduction in text entry error rates when tactile feedback was added to a mobile device touchscreen, but the keys there were much smaller, needing more accurate selection. A further experiment is needed to investigate this and find the target size at which tactile feedback starts to improve text entry accuracy.

Our second hypothesis was that the different body locations used to deliver the tactile feedback would produce differing speed and accuracy results. Again this is partially confirmed as we found that the wrist and upper arm lead to the fastest text entry rates, both being better than having no tactile feedback. It is not clear why these two locations performed the best, but is perhaps because they are the closest locations to the hand that is doing the typing. It may be easier to integrate the typing action with the tactile feedback when it is closer to the hand. However, in our earlier mobile study we found a strong effect for feedback on the device, but we did not find this in the present study. Further investigation is needed to understand these effects, but the results do show that distal tactile feedback presented via a watch, bracelet or armband would have a beneficial effect on text entry rates when typing on a tabletop computer.

\subsection{Comparison with our Previous Study}

We designed our experiment to be directly comparable with our previous study into mobile phone text entry [13] (where we compared text entry performance on a phone with a physical keyboard, a standard touchscreen phone and a touchscreen phone with added tactile feedback). We used the same code, experimental design and Tactons as before. The comparison reveals some interesting findings. A one-way between-subjects ANOVA on accuracy (we collapsed our conditions down to one as we saw no effect of accuracy in our experiment) showed a 
significant main effect for keyboard type $(F(4,55)=20.9$ $\mathrm{p}<0.001$ ), with our tabletop soft keyboard performing significantly more accurately than a standard touchscreen phone for text entry $(p<0.001)$. There were no differences between the other conditions (see Figure 11). This shows that our tabletop soft keyboard performed similarly to a mobile phone with a good keypad or tactile feedback. It suggests that a phone with only a touchscreen should not be used for standard qwertybased text entry on a tabletop computer, but other types of phones may allow input that is as accurate as the tabletop's keyboard. The phone keypads did not perform better than the touchscreen keyboard for accuracy, so would not give any direct benefit for text entry.

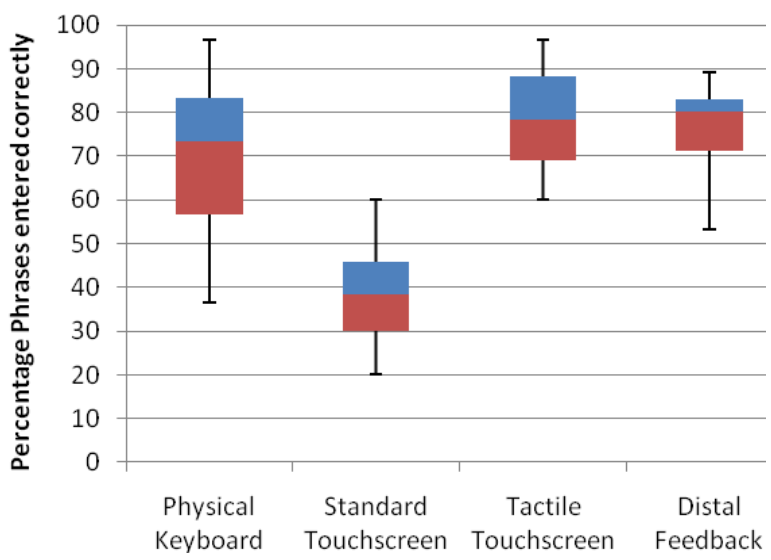

Figure 11: Comparing our results on text entry accuracy with those of our previous experiment [13]. The first three boxes show Hoggan's mobile phone results and the final box is the mean of our results.

Hinrichs [12] has suggested that a mobile device could be used for text entry on a tabletop computer (although did not present any strong evidence for this) and our results show that a mobile phone could be used for such a purpose, providing that it has either a physical keyboard or a soft keyboard with tactile feedback added. Many mobile phones are now becoming touchscreen only and doing away with a physical keypad (the Apple iPhone is a current example). It is therefore important to ensure that tactile feedback is used to get as good a text entry performance as possible. There are alternative ways of entering text on mobile phones, such as Shapewriter (www.shapewriter.com), that are designed to be faster to use than a standard touch keyboard, but it is not clear if these systems would see similar benefits from tactile feedback.

Using a phone for input on a tabletop computer has many other advantages. It would allow for private text entry by one user (passwords for example) and would also free up space on the tabletop display that would normally be needed to show the soft keyboard (especially useful if multiple users needed soft keyboards displayed at the same time). We are also planning to investigate a range of interactions with other types of widgets that are found on a tabletop computer. Work on mobile phones and desktop interfaces has found that tactile feedback can help in interaction with other types of widgets and we will investigate if this transfers to our phone/tabletop combination. We will also investigate the possibilities of audio feedback provided from the mobile phone, giving a private audio display.

In conclusion, the experiment presented here investigated if distal tactile feedback presented on a mobile phone could aid typing on a tabletop computer with a soft keyboard. Our results showed that in terms of Words per Minute tactile feedback presented to the wrist or upper arm made text entry significantly faster. This means that tactile feedback can be added to tabletop computers very simply by using the mobile phones that users carry with them when they come the table as personal tactile displays.

\section{ACKNOWLEDGMENTS}

This work was funded by an EPSRC DTA PhD scholarship.

\section{REFERENCES}

[1] Microsoft Surface, http://www.microsoft.com/SURFACE/index.html.

[2] Multitouch Cell, http://multitouch.fi/.

[3] NUI Horizon, http://naturalui.com/solutions/products.html.

[4] Brewster, S.A.: Overcoming the lack of screen space on mobile computers. Springer Verlag

[5] Brown, L.M., Brewster, S.A., Purchase, H.C.: Multidimensional tactons for non-visual information presentation in mobile devices. Proceedings of the 8th conference on Human-computer interaction with mobile devices and services. ACM, Helsinki, Finland (2006)

[6] Everitt, K.M., Forlines, C., Ryall, K., Shen, C.: Observations of a shared tabletop user study. Proc. CSCW 2005: Interactive Poster (2005)

[7] Forlines, C., Wigdor, D., Shen, C., Balakrishnan, R.: Direct-touch vs. mouse input for tabletop displays. Proceedings of the SIGCHI conference on Human factors in computing systems. ACM, San Jose, California, USA (2007)

[8] Han, J.Y.: Low-cost multi-touch sensing through frustrated total internal reflection. Proceedings of the 18th annual ACM symposium on User interface software and technology. ACM, Seattle, WA, USA (2005)

[9] Hancock, M.S., Shen, C., Forlines, C., Ryall, K.: Exploring non-speech auditory feedback at an interactive multiuser tabletop. Proceedings of Graphics Interface 2005. Canadian Human-Computer Communications Society, Victoria, British Columbia (2005)

[10] Hardy, R., Rukzio, E.: Touch $\backslash \&$ interact: touch-based interaction of mobile phones with displays. Proceedings of the 10th international conference on Human computer interaction with mobile devices and services. ACM, Amsterdam, The Netherlands (2008)

[11] Hart, S.G., Stavenland, L.E., Hancock, P.A., Meshkati, N.: Development of NASA-TLX (Task Load Index): Results of empirical and theoretical research. Human Mental Workload. Elsevier (1988) 139-183

[12] Hinrichs, U., Hancock, M., Collins, C., Carpendale, S.: Examination of Text-Entry Methods for Tabletop Displays. Horizontal Interactive Human-Computer Systems, 2007. TABLETOP '07. Second Annual IEEE International Workshop on (2007) 105-112

[13] Hoggan, E., Brewster, S.A., Johnston, J.: Investigating the effectiveness of tactile feedback for mobile touchscreens. Proceeding of the twenty-sixth annual SIGCHI conference on Human factors in computing systems. ACM, Florence, Italy (2008) 
[14] Ideum: $\mathrm{mt}^{\wedge} 2$ Table, http://www.ideum.com/products/multitouch.

[15] Ishii, H., Ullmer, B.: Tangible bits: towards seamless interfaces between people, bits and atoms. Proceedings of the SIGCHI conference on Human factors in computing systems. ACM, Atlanta, Georgia, United States (1997)

[16] Koskinen, E., Kaaresoja, T., Laitinen, P.: Feel-good touch: finding the most pleasant tactile feedback for a mobile touch screen button. Proceedings of the 10th international conference on Multimodal interfaces. ACM, Chania, Crete, Greece (2008)

[17] MacKenzie, I.S., Soukoreff, R.W.: Phrase sets for evaluating text entry techniques. $\mathrm{CHI}$ ' 03 extended abstracts on Human factors in computing systems. ACM, Ft. Lauderdale, Florida, USA (2003)

[18] Mackenzie, I.S., Zhang, S.X., Soukoreff, R.W.: Text entry using soft keyboards. Behaviour and Information Technology 18 (1999) 235-244

[19] Marshall, P., Hornecker, E., Morris, R., Sheep Dalton, N., Rogers, Y.: When the fingers do the talking: A study of group participation with varying constraints to a tabletop interface. Horizontal Interactive Human Computer Systems, 2008. TABLETOP 2008. 3rd IEEE International Workshop on (2008) 33-40

[20] McCarthy, J.F., Congleton, B., Maxwell Harper, F.: The context, content $\backslash \&$ community collage: sharing personal digital media in the physical workplace. Proceedings of the ACM 2008 conference on Computer supported cooperative work. ACM, San Diego, CA, USA (2008)

[21] Mortimer, B.J.P., Zets, G.A., Cholewiak, R.W.: Vibrotactile transduction and transducers. The Journal of the Acoustical Society of America 121 (2007) 2970-2977

[22] Poupyrev, I., Maruyama, S., Rekimoto, J.: Ambient touch: designing tactile interfaces for handheld devices. Proceedings of the 15th annual ACM symposium on User interface software and technology. ACM, Paris, France (2002)

[23] Ringel Morris, M., Morris, D., Winograd, T.: Individual audio channels with single display groupware: effects on communication and task strategy. Proceedings of the 2004 ACM conference on Computer supported cooperative work. ACM, Chicago, Illinois, USA (2004)
[24] Ryall, K., Forlines, C., Shen, C., Morris, M.R., Everitt, K.: Experiences with and Observations of Direct-Touch Tabletops. Proceedings of the First IEEE International Workshop on Horizontal Interactive Human-Computer Systems. IEEE Computer Society (2006)

[25] Scott, S.D., Sheelagh, M., Carpendale, T., Inkpen, K.M.: Territoriality in collaborative tabletop workspaces. Proceedings of the 2004 ACM conference on Computer supported cooperative work. ACM, Chicago, Illinois, USA (2004)

[26] Sears, A., Revis, D., Swatski, J., Crittenden, R., Shneiderman, B.: Investigating Touchscreen Typing: The Effect of Keyboard Size on Typing Speed. Behaviour and Information Technology 12 (1993) 17-22

[27] Silfverberg, M., MacKenzie, I.S., Kauppinen, T.: An isometric joystick as a pointing device for handheld information terminals. No description on Graphics interface 2001. Canadian Information Processing Society, Ottawa, Ontario, Canada (2001)

[28] Soukoreff, R.W., MacKenzie, I.S.: Metrics for text entry research: an evaluation of MSD and KSPC, and a new unified error metric. Proceedings of the SIGCHI conference on Human factors in computing systems. ACM, Ft. Lauderdale, Florida, USA (2003)

[29] Tikka, V., Laitinen, P.: Designing Haptic Feedback for Touch Display: Experimental Study of Perceived Intensity and Integration of Haptic and Audio. Haptic and Audio Interaction Design (2006) 36-44

[30] Tryhorn, C.: Developing countries drive explosion in global mobile phone use, http://www.guardian.co.uk/business/2009/mar/02/mobilephone-internet-developing-world. 2009 (2009)

[31] Tse, E., Histon, J., Scott, S.D., Greenberg, S.: Avoiding interference: how people use spatial separation and partitioning in SDG workspaces. Proceedings of the 2004 ACM conference on Computer supported cooperative work. ACM, Chicago, Illinois, USA (2004)

[32] Wigdor, D., Penn, G., Ryall, K., Esenther, A., Shen, C.: Living with a Tabletop: Analysis and Observations of Long Term Office Use of a Multi-Touch Table. Horizontal Interactive Human-Computer Systems, 2007. TABLETOP '07. Second Annual IEEE International Workshop on (2007) 60-67 